\title{
Suppression of Residual Single-Photon Absorption Relative to Two-Photon Absorption in High Finesse Planar Microcavities
}

\author{
Wei-Hua Guo, John O’Dowd, Edward Flood, Tom Quinlan, Michael Lynch, Ann Louise Bradley, \\ John Francis Donegan, Senior Member, IEEE, Krzysztof Bondarczuk, Paul J. Maguire, Member, IEEE, and \\ Liam P. Barry, Member, IEEE
}

\begin{abstract}
Suppression of residual single-photon absorption (SPA) relative to two-photon absorption (TPA) in a high finesse GaAs planar microcavity is explored. The TPA photocurrent becomes larger than the SPA photocurrent as long as the incident continuous-wave optical power exceeds $0.09 \mathrm{~mW}$. An optical power of $5 \mathrm{~mW}$ would be required if the relative SPA suppression did not exist.
\end{abstract}

Index Terms-Finesse, planar microcavity, single-photon absorption (SPA), two-photon absorption (TPA).

\section{INTRODUCTION}

$\mathbf{T}$ WO-PHOTON absorption (TPA) in semiconductors is of strong interest in applications such as optical sampling [1], optical clock recovery [2], and optical performance monitoring [3]. Although it is a weak third-order nonlinear process, TPA in semiconductors can be made very sensitive by employing absorption over a long distance [4], high gain in an avalanche photodiode [5], or high enhancement in a planar microcavity [6]. In TPA photodetectors, residual single-photon absorption (SPA) also exists due to deep defects or the Franz-Keldysh effect [7]. For optical signals with very low-repetition frequency and very high peak power, the SPA current accounts for so small a portion of the total current detected it can be neglected. However, as the optical signal repetition frequency becomes high and the peak power decreases, the influence of SPA can no longer be neglected because it may even exceed the TPA current. So addressing residual SPA is a very important issue if these TPA detectors are to be used in optical communications since optical signals used in this environment have relatively low peak powers $(\sim \mathrm{mW})$. In [8], it has been shown that the TPA efficiency can be enhanced greatly by

\footnotetext{
Manuscript received April 11, 2008; revised May 26, 2008. This work was supported by SFI under its CSET Centre for Telecommunication Value Driven Research (CTVR), Grant 03/CE3/I405, and its RFP programme, Grant RFP/ 2006/ENE012.

W.-H. Guo, J. O'Dowd, E. Flood, T. Quinlan, M. Lynch, A. L. Bradley, and J. F. Donegan are with the Semiconductor Photonics Group, School of Physics and Centre for Telecommunication Value Driven Research (CTVR), Trinity College, Dublin 2, Ireland (e-mail: guow @ tcd.ie).

K. Bondarczuk, P. J. Maguire, and L. P. Barry are with the Radio and Optical Communications Laboratory, School of Electronic Engineering, Dublin City University, Dublin 9, Ireland.

Color versions of one or more of the figures in this letter are available online at http://ieeexplore.ieee.org.

Digital Object Identifier 10.1109/LPT.2008.927886
}

using a high finesse planar microcavity. In this paper, we show that this high finesse microcavity not only improves the TPA efficiency but also suppresses the residual SPA relative to TPA so as to decrease the power requirement for TPA to dominate.

\section{THEORETICAL MODEL}

Without any cavity enhancement, the TPA current generated in a TPA photodetector can be generally described by $i_{\mathrm{TPA}}=$ $C \beta l e P_{\text {ave }}^{2} /\left(2 h \nu S d_{\text {gen }}\right)$, where $C$ is a factor related to the detector collection efficiency, $\beta$ is the TPA coefficient, $l$ is the absorption length, $S=\pi w^{2}$ is the spot area where $w$ is the beam waist of the incident beam assumed to be Gaussian, $e$ is the electron charge, $h \nu$ is the photon energy, and $d_{\text {gen }}$ is the generalized duty cycle of the incident optical signal [3]. The residual SPA current can be expressed as $i_{\mathrm{SPA}}=C \alpha l e P_{\text {ave }} /(h \nu)$, where $\alpha$ is the SPA coefficient. To have the TPA current larger than the SPA current requires $P_{\text {ave }}>2 \alpha S d_{\text {gen }} / \beta$. So reducing the spot size or reducing the generalized duty cycle can reduce the power requirement for TPA to dominate. If the absorption layer of the detector is placed between two highly reflective Bragg mirrors, the optical field can be greatly enhanced in the absorption layer so that TPA and SPA will both be enhanced. The TPA enhancement factor for the normally incident resonant wavelength is [9]

$$
\zeta_{\mathrm{TPA}}=\frac{\left(1-R_{1}\right)^{2}\left(1+R_{2}^{2}+4 R_{2}\right)}{\left(1-\sqrt{R_{1} R_{2}}\right)^{4}}
$$

where $R_{1}$ and $R_{2}$ are the top and bottom mirror reflectivity, respectively. The SPA enhancement factor is

$$
\zeta_{\mathrm{SPA}}=\frac{\left(1-R_{1}\right)\left(1+R_{2}\right)}{\left(1-\sqrt{R_{1} R_{2}}\right)^{2}} .
$$

To obtain these two formulae we have assumed that the microcavity structure is simply a $1-\lambda$-thick absorption layer sandwiched by two Bragg mirrors, as in [8]. The TPA enhancement is larger than the SPA enhancement as it depends on the squared optical intensity inside the absorption layer. Although SPA is enhanced as compared with the noncavity case, relative to TPA, it is in fact relatively suppressed. So now the power required for the TPA current to exceed the SPA current can be expressed as

$$
P_{\text {ave }}>\frac{2 \alpha S d_{\text {gen }}}{\beta} \frac{\zeta_{\mathrm{SPA}}}{\zeta_{\mathrm{TPA}}} \equiv \frac{2 \alpha S d_{\text {gen }}}{\beta} \frac{1}{\xi}
$$




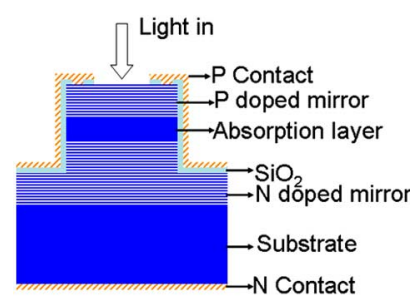

(a)

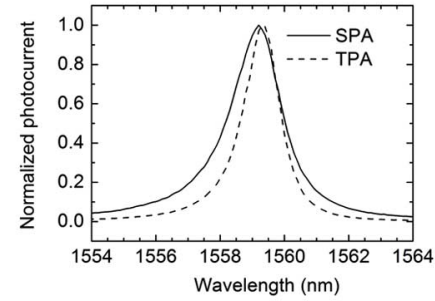

(b)
Fig. 1. (a) Schematic diagram of the device structure; (b) Cavity spectrum measured as the TPA and SPA dominated respectively and the incident beam was nearly a parallel beam.

where $\xi$ describes the relative suppression of the residual SPA due to the high finesse microcavity. According to the following analysis, $\xi$ can reach a level around 100 .

\section{EXPERIMENT}

The TPA detector is a GaAs planar microcavity structure as shown in Fig. 1(a). The top mirror is p-doped and consists of 13-pairs of GaAs-AlAs layers. The bottom mirror is n-doped and consists of 23-pairs of GaAs-AlAs layers. The absorption layer is $0.46-\mu \mathrm{m}$-thick $(1 \lambda)$ unintentionally doped GaAs. The resonant wavelength is designed to be $1560 \mathrm{~nm}$. The detector is top and bottom contacted with an input aperture, of $20 \mu \mathrm{m}$ diameter, on the top. The experimental setup is as follows: the continuous-wave $(\mathrm{CW})$ output from a widely tunable laser passes through an erbium-doped fiber amplifier, followed by an attenuator and then a polarization controller. It is focused onto the detector through a lensed fiber. The photocurrent is detected by a Keithley picoameter.

Because TPA in GaAs is polarization-sensitive [10], the polarization controller is used to adjust the incident polarization so as to maximize the photocurrent. The optimized polarization is kept unchanged throughout the measurement. The cavity spectrum is first measured using a defocused spot much larger than the detector aperture. In this case, the input Gaussian beam can be treated as a parallel beam. The spectrum is measured by scanning the tunable laser wavelength while keeping the incident power constant. The incident power is selected to ensure either TPA $(5.84 \mathrm{~mW})$ or SPA $(0.2 \mathrm{~mW})$ dominates the detector response. Fig. 1(b) shows the normalized spectrum. The full-width at half-maximum of the SPA spectrum is $1.88 \mathrm{~nm}$, compared with $1.32 \mathrm{~nm}$ for the TPA spectrum. The TPA spectral width is exactly 1.4 times less than the SPA spectral width, which is expected because the TPA depends on the squared optical intensity inside the cavity while the SPA signal just depends on the optical intensity. The SPA spectral width is broader than our design value, which could be caused by factors like absorption in the doped mirrors, especially the p-doped mirrors, and also layer thickness fluctuations. However, through simulation we find that the penetration depth of the optical field into the mirrors is not strongly influenced by these factors, so we assume that the practical effective cavity length is still our design value, which is $2.04 \mu \mathrm{m}$. From the SPA spectral width we can find the averaged mirror reflectivity $R=\left(R_{1} R_{2}\right)^{1 / 2}$ to be 0.965 . As seen from (1) and (2), even as the averaged mirror reflectivity is fixed, the distribution of the top and bottom mirror reflectivity

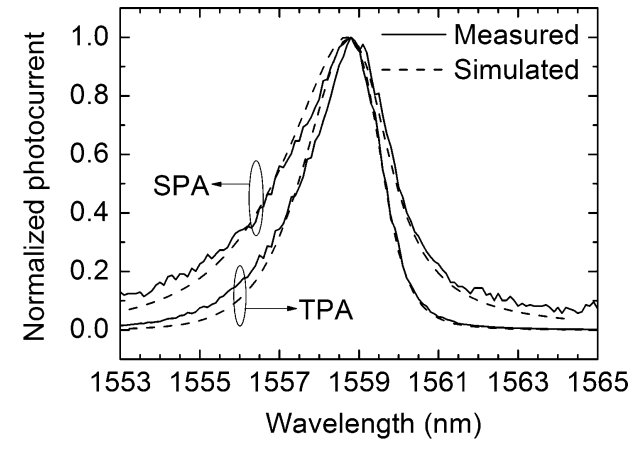

Fig. 2. Cavity spectrum measured and simulated with the TPA and SPA dominating respectively and a tightly focused incident beam.

will still influence the final enhancement. A higher bottom reflectivity tends to yield higher enhancement. In our case, the bottom mirror is designed to generate a reflectivity as close as possible to one, so it has a much larger number of periods than the top mirror. Practically, because the absorption loss in the p-doped top mirror would be much higher than in the n-doped bottom mirror, we would expect that the bottom mirror reflectivity is closer to our design value. So we estimate our bottom mirror reflectivity would be between 0.98 and 0.99 . We take a value of 0.985 here, so the top mirror reflectivity would then be about 0.945 . Based on these estimations, the TPA enhancement is 11600 and the SPA enhancement is 90 as calculated from (1) and (2). The SPA suppression factor $\xi$ would be around $130 \mathrm{ac}$ cording to (3).

However practically we need to consider the limited acceptance angle of the planar microcavity which is defined by the angle at which the intensity inside the cavity drops by half as the incident beam is angled from normal incidence to greater angles. The incident wavelength is the resonant wavelength for the normal incidence case. The acceptance angle which is directly related to the cavity spectral width is estimated to be $12.7^{\circ}$. The lensed fiber we use can generate a Gaussian beam with a beam waist of approximately $3.5 \mu \mathrm{m}\left(1 / e^{2}\right)$ which corresponds to a divergence angle of $16.1^{\circ}$. We measured the spectrum again with the beam tightly focused, giving a spot on the detector much smaller than the detector aperture. In this case, the incident power used for the TPA and SPA dominance is $1.3 \mathrm{~mW}$ and $4.6 \mu \mathrm{W}$, respectively. The result is shown in Fig. 2. A clear asymmetry can be seen which is due to the angular components of the focused Gaussian beam. A theoretical analysis similar to [9] taking account of the greater angular distribution is carried out, with the result comparing well with the experimental result as shown in Fig. 2. Because of the limited acceptance angle of the planar microcavity, the angled components of the incident focused Gaussian beam cannot be enhanced as much as the normally incident component, so the overall enhancement would drop. From the same simulation, the decrease in the enhancement for TPA is found to be $71 \%$ compared with $32 \%$ for SPA.

With the incident wavelength corresponding to the peak wavelength from the spectrum in Fig. 2, we measure the photocurrent as a function of the $\mathrm{CW}$ power. The result is shown in Fig. 3. A fitting of the current-power curve yields a relation of $i(\mathrm{nA})=3.70 P^{2}(\mathrm{~mW})+0.33 P(\mathrm{~mW})$ which means for 


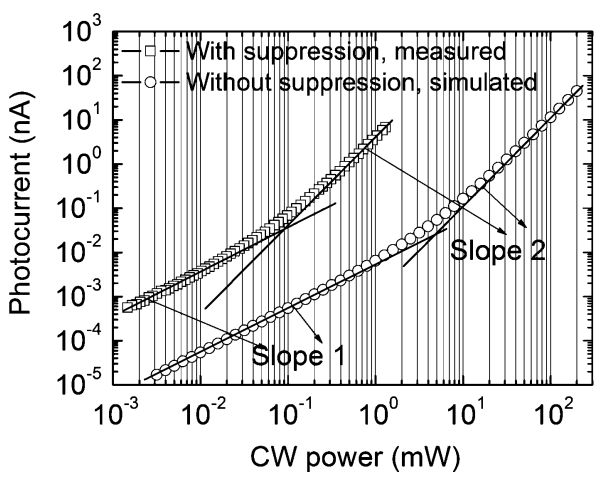

Fig. 3. Measured photocurrent as the incident $\mathrm{CW}$ power is varied. The curve without SPA suppression was simulated from a noncavity detector with the same absorption length.

an input power equal to $0.09 \mathrm{~mW}$, the TPA current is equal to the SPA current. Since reverse bias does not increase the photocurrent detected, we do not apply any reverse bias in our measurement and the dark current is lower than $1 \mathrm{pA}$. Now using the formula (1) with the decrease in enhancement taken into account, the TPA coefficient in our case would be $14.6 \mathrm{~cm} / \mathrm{GW}$ assuming the collection efficiency is equal to one. In [10], the measured TPA coefficient of (001)-GaAs at $950 \mathrm{~nm}$ is between 20 and $27 \mathrm{~cm} / \mathrm{GW}$, where $20 \mathrm{~cm} / \mathrm{GW}$ is for linear polarization along the (100) direction which describes the strength of the TPA in the isotropic limit [11], and $27 \mathrm{~cm} / \mathrm{GW}$ is for linear polarization along the (110) direction which represents an anisotropic factor of -0.76 . The wavelength dependence of the TPA coefficient is described by the function [12] $F\left(2 h \nu E_{g}^{-1}\right)=\left(2 h \nu E_{g}^{-1}-1\right)^{3 / 2}\left(2 h \nu E_{g}^{-1}\right)^{-5}$, which was obtained based on an isotropic band structure. Using this relation we would estimate the TPA coefficient in the isotropic limit in (001)-GaAs at $1560 \mathrm{~nm}$ is $12.5 \mathrm{~cm} / \mathrm{GW}$. The anisotropic factor at $1560 \mathrm{~nm}$ was estimated to be half of the value at $950 \mathrm{~nm}$ [11], so a value of -0.38 could be used. Then the TPA coefficient for polarized light along the (110) direction at $1560 \mathrm{~nm}$ would be $14.8 \mathrm{~cm} / \mathrm{GW}$ which is also the highest TPA coefficient for any polarizations for (001)-GaAs. The value estimated from our measurement agrees with this value very well. In [13], a value around $19 \mathrm{~cm} / \mathrm{GW}$ was measured at $1560 \mathrm{~nm}$ which agrees reasonably well with our result taking account of their data fluctuation around $1550 \mathrm{~nm}$. From the measured SPA current, we estimate the SPA coefficient in our detector to be $0.93 \times 10^{-4} \mathrm{~cm}^{-1}$, which is a very low value. However, if there was no relative suppression of this small residual SPA, the SPA current would exceed the TPA current for the incident $\mathrm{CW}$ power less than $5 \mathrm{~mW}$ as shown in Fig. 3 where the simulated data is based on a noncavity detector with the same absorption length. If the acceptance angle of the microcavity could be increased allowing the focused beam to be enhanced as much as the nonfocused beam, the TPA signal would become equal to the SPA signal for an incident $\mathrm{CW}$ power of only $38 \mu \mathrm{W}$.

\section{CONCLUSION}

A high-finesse microcavity not only improves the TPA efficiency but also relatively suppresses the residual SPA resulting in a TPA dominated signal at much lower incident power. The TPA coefficient is estimated to be $\sim 15 \mathrm{~cm} / \mathrm{GW}$ at $1560 \mathrm{~nm}$ in (001) GaAs and the residual SPA coefficient in our unintentionally doped GaAs grown by metal-organic chemical vapor deposition is approximately $1.0 \times 10^{-4} \mathrm{~cm}^{-1}$.

\section{ACKNOWLEDGMENT}

The authors would like to thank D. Kilper and M. Dinu of Bell Laboratories, Crawford Hill, for valuable discussions.

\section{REFERENCES}

[1] K. Kikuchi, "Optical sampling system at $1.5 \mu \mathrm{m}$ using two photon absorption in Si avalanche photodiode," Electron. Lett., vol. 34, no. 13, pp. 1354-1355, 1998.

[2] R. Salem and T. E. Murphy, "Broad-band optical clock recovery system using two-photon absorption," IEEE Photon. Technol. Lett., vol. 16, no. 9, pp. 2141-2143, Sep. 2004.

[3] S. Wielandy, M. Fishteyn, and B. Zhu, "Optical performance monitoring using nonlinear detection,” J. Lightw. Technol., vol. 22, no. 3, pp. 784-793, Mar. 2004

[4] F. R. Laughton, J. H. Marsh, and A. H. Kean, "Very sensitive two photon absorption GaAs/AlGaAs waveguide detector for an autocorrelator," Electron. Lett., vol. 28, no. 17, pp. 1663-1665, 1992.

[5] C. Xu, J. M. Roth, W. H. Knox, and K. Bergman, "Ultra-sensitive autocorrelation of $1.5 \mu \mathrm{m}$ light with single photon counting silicon avalanche photodiode," Electron. Lett., vol. 38, no. 1, pp. 86-88, 2002.

[6] H. Folliot, M. Lynch, L. P. Barry, A. L. Bradley, L. A. Dunbar, J. Hegarty, J. F. Donegan, J. S. Roberts, and G. Hill, “Two-photon absorption photocurrent enhancement in bulk AlGaAs semiconductor microcavities," Appl. Phys. Lett., vol. 80, no. 8, pp. 1328-1330, 2002.

[7] M. M. Karkhanehchi, D. A. Barrow, A. C. Bryce, C. J. Hamilton, and J. H. Marsh, "The influence of single-photon absorption on the performance of the two-photon waveguide autocorrelator," IEEE J. Quantum Electron., vol. 33, no. 6, pp. 933-937, Jun. 1997.

[8] T. Krug, M. Lynch, A. L. Bradley, J. F. Donegan, L. P. Barry, H. Folliot, J. S. Roberts, and G. Hill, "High-sensitivity two-photon absorption microcavity autocorrelator," IEEE Photon. Technol. Lett., vol. 16, no. 6 , pp. 1543-1545, Jun. 2004.

[9] W. H. Guo, J. O’Dowd, M. Lynch, A. L. Bradley, J. F. Donegan, and L. P. Barry, "Influence of cavity lifetime on high-finesse microcavity two-photon absorption photodetectors," IEEE Photon. Technol. Lett., vol. 19, no. 6, pp. 432-434, Mar. 15, 2007.

[10] M. D. Dvorak, W. A. Schroeder, D. R. Andersen, A. L. Smirl, and B. S. Wherrett, "Measurement of anisotropy of two-photon absorption coefficients in zincblende semiconductors," IEEE J. Quantum Electron., vol. 30, no. 2, pp. 256-268, Feb. 1994.

[11] D. C. Hutchings and B. S. Wherrett, "Theory of anisotropy of twophoton absorption in zinc-blende semiconductors," Phys. Rev. B, vol. 49, no. 4, pp. 2418-2426, 1994.

[12] E. W. V. Stryland, M. A. Woodall, H. Vanherzeele, and M. J. Soileau, "Energy bandgap dependence of two-photon absorption," Opt. Lett., vol. 10, no. 10, pp. 490-492, 1985.

[13] A. Villeneuve, C. C. Yang, G. I. Stegeman, C. N. Ironside, G. Scelsi, and R. M. Osgood, "Nonlinear absorption in a GaAs waveguide just above half the band gap," IEEE J. Quantum Electron., vol. 30, no. 5, pp. 1172-1175, May 1994. 